\title{
Aspectos bioéticos en la Reforma de la Atención de Salud en Chile. II. Discriminación, libre elección y consentimiento informado
}

\author{
Dr. Eduardo Rosselot J. \\ Bioethical Aspects of Health Care \\ Reform in Chile. II. Discrimination, \\ free election and informed consent
}

Bioethical issues emerge each time health care reform projects are discussed. These affect diverse moral values and principles and have an impact on cultural, social and political areas. Thus, they demand more than just organizational, financial or administrative solutions. This review analyses discrimination, free election of professionals and informed consent. All three concepts are alluded in the legislative debate raised upon the actual process for health reform. Having clear ideas about these subjects is crucial to foresee the reactions expected to arise among physicians and the general public, when confronting the proposed changes (Rev Méd Chile 2003; 131: 1329-36).

(Key Words: Ethics, medical; Health care economics and administration; Health care reform)

Recibido el 21 de julio, 2003. Aceptado el 26 de agosto, 2003.

Departamento de Bioética y Humanidades Médicas, Departamento de Educación en Ciencias de la Salud, Facultad de Medicina, Universidad de Chile.

$\mathrm{E}^{\mathrm{n}}$ las propuestas de reforma de la atención de salud que se han gestado en el país y han sido motivo de debate público y legislativo ${ }^{1-7}$, dos temas tienen áreas comunes en las que existen severas discrepancias, dependientes de las posiciones con que se asumen o interpretan los principios bioéticos clásicos ${ }^{8}$.

El primer tema dice relación con las restricciones o favorecimientos a que arbitrariamente pueden estar sometidas las personas en la atención de salud, conculcándose por ello la equidad y exponiendo a maleficencia a quienes debieran ser los beneficiados. El segundo se refiere al consenti- miento informado, concepto relacionado básicamente con el principio de autonomía que aún se esfuerza por lograr preeminencia en un ambiente como el latinoamericano, todavía absorto en la tradición y el paternalismo. Con ellos no se agota la problemática bioética de la reforma de salud, múltiple pero común a todos estos procesos ${ }^{9-11}$, que cobran progresiva universalidad y preeminencia por las características de los servicios en materia de salud y por la forma de otorgar las prestaciones médicas, afectadas por diversas contingencias que dan pábulo a variadas transgresiones de los cuatro principios. 
Estos conceptos expresan planteamientos que rompen con la posición protagónica e incuestionada del profesional, que ha sido el paradigma en la medicina clásica, y que tiende a ser superada por una actitud real de servicio y una resuelta disposición a rendir cuenta de propósitos y acciones, evaluables con parámetros conocidos y precisos indicadores de satisfacción y cumplimiento.

De alguna manera, el énfasis con que estos denominadores sitúan al paciente en el centro del diálogo asistencial, reflejan la adopción de una nueva cultura en la medicina, el rescate de un comportamiento profesional que se ha desvirtuado por prejuicios, la modernidad y el mercado, y la clave para dar respuesta a un sentido más coherente de los cuidados de la salud. Estas apreciaciones justifican que ahondemos en la significación y trascendencia de los aspectos valóricos que, hoy día, se estiman indispensables y centrales en la atención de la salud humana.

\section{DisCRIMINACIÓN}

La igualdad de oportunidades en la atención de salud es quizá la expresión más palmaria de cómo se manifiestan y reclaman los derechos a la salud de las personas ${ }^{9-12}$. Todas las actas o declaraciones oficiales de organismos internacionales, de instancias legislativas o de instituciones que dan cabida a tales disposiciones definen la equidad como el valor fundamental para conformar esa garantía, sin excepción de "raza, condición socioeconómica, credos, género, nivel educacional"3, etc. Sin embargo, al implementar acciones para satisfacer determinadas exigencias no hay modelo que pueda sustraerse de hacer, de alguna manera, discriminaciones* que suscitan reparos sobre la justicia o transparencia de tales opciones. La causa no es otra que la lógica limitación de recursos ante demandas sanitarias que exceden las disponibilidades ${ }^{13}$.

Una forma común de discriminar en la atención frente a las demandas de salud, es lo que se entiende como priorización por patologías, méto- do propuesto en el modelo del Plan de Acceso Universal y Garantías Explícitas (AUGE), y que define cuáles afecciones y en qué condiciones deben ser objeto de trato preferencial y, supuestamente, garantizado. Sin duda que aceptar estas prioridades depende de la idoneidad y confiabilidad de quienes establezcan las categorías a privilegiar. Tanto más se afianzará la normativa cuanto mayores alcances y amplitud tenga la nómina de prestaciones, al cubrir un mayor número de susceptibles, y habrá menor sensación de injusticia o marginación por parte de quienes queden fuera de esa lista favorecida por "garantías explícitas". Resulta más fácil aceptar dicho predicamento, si se tiene el mayor grado de certeza posible de que no han jugado arbitrariedades ni ha habido exclusiones por condiciones étnicas, sociales, económicas, educacionales, religiosas, políticas u otras, ajenas a una razón propiamente médica o sanitaria ${ }^{8,12-14}$.

Es natural que se susciten reacciones contrarias a cualquier racionamiento de servicios, a cuyo usufructo se supone que existe un derecho universal. Expresan el sentimiento de ver vulnerada la igualdad original ${ }^{13}$, infringida la ética mínima ${ }^{14}$, atropellados los derechos de las personas ${ }^{15,16}$. Para atenuar eventuales transgresiones a principios éticos elementales, cuando son forzadas por causas insuperables, y sólo para evitar acciones de mayor maleficencia y en consideración al bien común -como en situaciones de emergencia ${ }^{18}$, podría invocarse razonablemente la solidaridad social, para que los menos expuestos a daño renuncien transitoriamente al derecho virtual a atenciones específicas en salud.

Cuando las políticas sanitarias han destacado determinados programas de atención, esto ha representado sólo decisiones técnicas de privilegiar ciertas acciones consideradas de mayor efectividad, eficiencia y eficacia ${ }^{1,15,19}$. Tales parámetros socio-económicos son legítimos para ponderar las decisiones a tomar, si existen limitaciones a las prestaciones sanitarias o hay que elegir entre alternativas programáticas, que no se pueden realizar conjuntamente. Esto es frecuente

*Discriminar se toma aquí en su acepción más amplia de diferenciar, distinguir una cosa de otra, sin que haya el menor prejuicio para menospreciar cualquiera de los elementos comparados en razón de su propio ser. En salud este diferenciar sólo puede suponer beneficencia con absoluto respeto a la justicia y no maleficencia. 
en la implementación de los programas de salud en el mundo, y en nuestro país lleva décadas de desarrollo sin que provoque reacciones desproporcionadas. Cuando su ejercicio ha sido pertinente, han traducido razones epidemiológicas y de costo y no una caprichosa discriminación ${ }^{20,21}$. La alternativa de cambiar las prestaciones de la canasta AUGE por programas específicos de atención prioritaria, que cubrieran diversos aspectos de cada patología seleccionada (desde la pesquisa y prevención hasta la terapia y rehabilitación, en cuanto fueran pertinentes), quizás habría generado menos rechazo que la proposición de lo que se denomina eufemísticamente "prestaciones garantizadas", en vez de canasta básica de prestaciones.

Hay que tener cuidado, por consiguiente, de no confundir discriminación en su sentido de desfavorecimiento, con la adopción de medidas de selección de grupos de usuarios, pacientes 0 , incluso, prestadores, por razones técnicas, de naturaleza epidemiológica, para lograr mayor beneficio social o impedir daños eventuales prevenibles. Por ejemplo, se ha destacado recientemente en la prensa nacional el reclamo de grupos homosexuales por medidas que ellos consideran discriminatorias de género, al normarse en la donación de sangre para los bancos de transfusión en los Servicios de Salud, el requerimiento de declarar abstinencia de relaciones homosexuales sin uso de condón. Sin perjuicio de discutir el uso, o no, del preservativo como elemento de seguridad en estos casos, así como la confiabilidad de una declaración expresa, sin duda que el restringir las donaciones de sangre de un grupo que se sabe tiene alto riesgo de ser portador de VIH y, por lo tanto, susceptible de transmitir desaprensivamente la enfermedad, no puede ser considerado arbitrario ni que atente contra la ética, por discriminación. Es cierto que la más adecuada información y razonada persuasión a los grupos que se auto consideran discriminados, es una medida de prudencia y de respeto a su autonomía que debiera primar, en el convencimiento de que las motivaciones epidemiológicas, sustentadas en la experiencia universal sobre procesos de alta contagiosidad, debieran ser suficientes para aceptar la política sanitaria a este respecto ${ }^{22-24}$.

\section{LIBRE ELECCIÓN DE LOS PROFESIONALES}

Vecino al concepto de discriminación, se puede abordar la idea de la restricción a la libre elección de los profesionales, en cuanto limitaría la autonomía. Por ende, esta facultad de los usuarios pareciera constituir una reivindicación sostenida fundamentalmente por éstos, como un derecho a decidir quién los asiste en la enfermedad, según la confianza, la información sobre sus capacidades, aptitudes y atributos, en tanto el paciente lo estime esencial para la relación profesional que se debe establecer. Superficialmente, tal aspiración no merece reparos, pero es fácil percibir que, desde hace mucho tiempo, ésta es una pretensión más vigente en el médico que en el paciente $y$, probablemente, más promovida en instancias gremiales que en el ámbito propiamente personal ${ }^{23}$.

La evolución experimentada por la estructura organizacional en la cual se ejerce la medicina, sea en el sector de atención privada 0 pública, ha hecho que la misma haya ido dejando de tener carácter individual, para transformarse progresivamente en institucional o de equipo. Por lo tanto, si cabe la posibilidad de elegir proveedor, será referida al organismo donde se da la atención y, tal vez como algo excepcional, a un profesional específico. Más aún tratándose de especialistas, lo habitual es que la atención sea propuesta por un tratante para un colega determinado, sólo excepcionalmente por la elección exclusiva del paciente. Esa conducta que trata de abogar por una libre elección primordial, del médico por el paciente, aparte de ser inaplicable en la medicina moderna -querámoslo o no, institucionalizada- sólo es factible en un régimen de libre mercado en el ejercicio profesional aunque, curiosamente, quienes propician esa doctrina son los más ardientes defensores del Estado, como colectivo proveedor de las acciones de salud. En todo caso, ese tipo de libre elección no puede ir sino que en franca desaparición en tanto la medicina se desarrolla como servicio, con responsabilidad compartida por todo el equipo de salud, cualquiera sea el propietario de su organización, y limitándose solamente a quienes tienen alta capacidad de pago para comprar prestaciones particulares. 
La calidad de la atención es lo que debiera buscarse como central en el establecimiento de la relación a convenir entre paciente y médico. Por lo tanto, esa y no otra debiera constituir la razón fundamental de una eventual elección. Si esta característica del trabajo médico, que debe ser expresión de su profesionalismo ${ }^{26,27}$, queda resguardada en su formación, no debiera haber motivo para pretender como un derecho esa particular, utópica y verdaderamente anacrónica, libre elección.

Por último, no hay razones para presumir que la relación médico-paciente originada en la libre elección del primero por el segundo, tenga más valor ético que la predominante en la actualidad. $\mathrm{Si}$ es cierto que la relación tradicional estaba mayormente definida por una actitud paternalista del profesional, mientras en la actual predominan los valores de calidad técnica, decisión informada y confirmación de la propia autonomía ${ }^{21,26,27}$, se puede suponer que, existiendo en ambos modelos la posibilidad de desviaciones aberrantes, es la última la más consonante con el progreso médico y con los cambios que el ejercicio profesional y la mayor conciencia de su responsabilidad de usuarios, ha determinado en los pacientes.

En cualquier caso, lo importante es que si hay elección lo sea efectivamente; es decir, que constituya una opción a tomar entre alternativas igualmente idóneas y pertinentes respecto a la situación que enfrenta el paciente, a los recursos disponibles, a los costos involucrados (financieros, físicos, morales, sociales o de cualquiera otra índole valórica) y a cualquier otra eventual consecuencia de esa decisión. Toda presión, fuerza 0 influencia que no se concilie con la libre determinación del paciente, descalifica la opción desde el punto de vista de su propiedad y legítimo mejor interés. Respecto a la influencia de un mismo profesional en la toma de decisiones, que incluyan derivaciones o exclusiones no basadas en criterios exclusivamente técnicos, se pueden establecer situaciones de grave responsabilidad por conflictos de interés, que deben ser previstos y cautelados ${ }^{28-30}$.

Merece mencionarse, también, la desorientación que puede generar, en un medio regido y rendido al mercado 31 , la publicidad y la promoción de servicios médicos individuales, de grupos de especialistas o de instituciones de salud. Movidos por objetivos predominantes de lucro, de sobrevivencia en una atmósfera competitivamente despiadada o de sobre exposición, para hacer relevantes su potencialidad o logros efectivos en una medicina de acelerado progreso, a menudo se presentan con los instrumentos que maneja habitualmente el comercio, para destacar sus productos. No olvidemos que hay amplia distancia entre los valores empresariales y los valores profesionales $^{32}$, lo que puede determinar no sólo estilos diferentes para llegar a los destinatarios de los respectivos actos, sino que actitudes no reconciliables para informarlos y orientarlos en la elección de quiénes, para qué y cómo llevar a cabo la acción sanitaria requerida $13,33,34$.

Vale la pena señalar, por último, que en la aplicación global del modelo de atención ambulatoria, que supone el nivel primario como puerta de entrada al sistema, y que se sustenta en apreciaciones de costo efectividad, rendimiento y máximo aprovechamiento de los recursos profesionales y técnicos para una racional atención, el acceso a los cuidados integrados tiene que ser focalizado en determinados especialistas y lugares de atención ${ }^{13,31-38}$. Ello no puede ser considerado coercitivo ni atentatorio al libre acceso al sistema sino, más bien, como una racionalización que busca las mejores alternativas para una atención del mejor nivel y rendimiento. Si en este proceso se privilegia la efectividad y eficiencia de la atención de salud, no puede invocarse transgresiones éticas a la libre elección, por no constituir ésta un valor absoluto o superior, ni que está abrogado por el sistema. La libre elección es un valor que puede ser considerado beneficiente y tendría, en la relación médico paciente, que subordinarse al bien común que establece el derecho a una atención de excelencia de todos los pacientes, o del mayor número ${ }^{39}$, por los profesionales. En la estructura organizacional de los sistemas de salud modernos, el procurar una atención equitativa y universal puede entrar en conflicto con la selectividad de la libre elección y, en tal caso, las decisiones pertinentes condicionan los derechos imperfectos a los perfectos, los principios privados a los públicos, la ética de máximos a la ética de mínimos ${ }^{40}$. 


\section{CONSENTIMIENTO INFORMADO}

Los derechos y deberes de los pacientes han estado cobrando identidad en el ámbito normativo $^{41}$ y legislativo ${ }^{3}$ en nuestro país, en el último quinquenio. Son demostración de la importancia que alcanza el reconocimiento y el ejercicio de una autonomía responsable en la satisfacción de los requerimientos de salud de las personas. Entre las disposiciones más trascendentes incluidas en el proyecto de reforma de la salud nacional a que estamos refiriéndonos, están las relativas al consentimiento informado.

Al consentimiento informado se le conocen dos connotaciones bastante dispares según nos atengamos a su significado instrumental o al concepto socio cultural subyacente a su irrupción en la problemática de las decisiones sobre los cuidados de salud. En nuestro medio, un instrumento común por el que el paciente autoriza al médico a realizar una intervención profesional, han sido los formularios de clínicas y hospitales que los enfermos han firmado para avalar este permiso. El profesional ha confiado en que este documento le proporcionaría suficiente indemnidad frente a eventuales demandas del paciente por complicaciones o mala práctica en el ejercicio del acto médico. Se ha supuesto que bastaba un relativo conocimiento del beneficiario y que aceptara los procedimientos a aplicar, para desestimar cualquier queja posterior sobre eventuales consecuencias de los mismos. El énfasis ha estado puesto en el consentir, de algún modo equivalente a asumir la responsabilidad por lo que hubiera de suceder por obra de lo consentido. Para efectos prácticos, este elemento pasó a ser una constancia protectora, tanto para el médico como para el paciente frente a posibles daños derivados de un acto médico preciso, que aun careciendo de efectos legales daba un marco para la intervención, susceptible de hacerse instrumental ante reclamos o $\operatorname{cargos}^{42,43}$. En cierto modo, esta versión falaz del consentimiento, ha sido un impedimento para que el real sentido del proceso de consentimiento informado se incorpore al trabajo habitual de los profesionales de la salud y contribuya a afianzar la relación clínica, como se pretende de su más apropiada concepción.
El consentimiento informado constituye la explicitación más clara del respeto a la autonomía y del afán de darle debida consideración. En tal sentido, al desafiar al paternalismo, este concepto ha sido considerado transgresor de privilegios ancestrales que el arte médico ha mantenido bajo diversas culturas y que permanecen acendrados en quienes todavía ven en el doctor la suma de virtudes y conocimientos que sólo él puede ejercer con apropiado dominio y autoridad. Sin embargo, esa insostenible pretensión ha sido debilitada tras experiencias como las de Nuremberg y Tuskegee, la irrupción tecnológica en la medicina y el despertar de la bioética, con el reconocimiento de que los derechos civiles tienen ingerencia en los cuidados de la salud y que la responsabilidad de su salvaguarda reside, fundamentalmente, en el paciente ${ }^{42-46}$. Por supuesto que la necesidad de generalizar el consentimiento informado con esta connotación, no es reconocida universalmente y, menos, una segunda apreciación valórica sobre su irremplazable función como factor que, a la vez, perfecciona los nexos de una relación médico paciente efectiva y surge, coherentemente, en base a esa misma ligazón. Sin embargo, así se pasa a configurar un proceso de interacción en que la decisión, traducida en consentimiento, surge fluidamente del acompañamiento que significa la relación médico paciente, como un acto comunicacional de hondo contenido y firme continuidad, y donde ese vínculo, motivado pero no exclusivamente dedicado a la enfermedad sino que al paciente, hace adulta una relación que "tradicionalmente tuvo el peligro de infantilizar al enfermo y endiosar al médico"43.

Esta deformada disposición frente a la enfermedad, como suele suceder bajo un clima de temor con esperanza, mezclado a expectación confiada en la infalibilidad, en el mejor escenario de una relación paternalística, merece ser cambiada incluso como medio esencial para apoyar los cuidados para la salud, y ése es un esfuerzo al que la doctrina del consentimiento informado puede contribuir resueltamente.

Meisel y Kuczewski ${ }^{47}$ resumen en 8, los mitos que han dificultado la aceptación de este encuentro ineludible con positivas proyecciones, que debiera concluir siempre en un acuerdo de atención más que en una aceptación resignada. $\mathrm{Al}$ 
mismo tiempo, establecen las diferencias entre un acto formal, para cumplir sólo una obligación de prevención, y un proceso enriquecido y enriquecedor de una relación entre un profesional, comprometido por sus acciones y por su afecto, y quien busca confiado y esperanzado su mejoría 0 su alivio. No es igual, firmar un consentimiento que decidir luego de tener una acabada información. Un documento rutinario, podrá referir que hubo un acto circunstancial de transmisión de información, pero nada dice del grado de comprensión, seguridad y tranquilidad, ni de la responsabilidad compartida en el manejo de la enfermedad. El consentimiento no es simplemente una señal de advertencia sobre los riesgos de las intervenciones programadas, que releva al médico de dar mayor información. Son las opciones terapéuticas y sus expectativas las importantes de referir, de tal modo que el paciente pueda aclarar sus dudas y resolver entre diversas alternativas, si es que existen 0 , incluso, optar por ninguna. Tampoco debe tomarse la información prevista como una oferta abierta para que el paciente elija según su mejor saber y entender. Si hay una responsabilidad indelegable del médico es su calidad de consejero. Ello no implica que el médico decida en lugar del paciente sino que abre el proceso a una interacción, para compartir y colaborar en una decisión conjunta y, sobre todo, pensando en la evolución muchas veces compleja y con sucesivas contingencias de la enfermedad, atenta a sus variaciones y a los necesarios e inevitables cambios de perspectiva.

Un mito que, en este contexto, provoca justa reticencia es que el paciente debe ser informado de todo. Lo cierto es que no procede ni más ni menos que lo estimado razonable por el médico y por el paciente. Ésta, aunque vaga aseveración, es respaldada porque se supone un cabal conocimiento del paciente por el médico, razón demás para adscribir este acto a un proceso y no a una contingencia puntual. Por eso es que también existen, situaciones eximentes del consentimiento, como son las urgencias vitales y la incapacidad del paciente para establecer una comunicación efectiva, que garantice la comprensión de lo que debiera proceder, estipulándose en su reemplazo la comparencia de representantes, no sólo para cubrir aspectos legales sino para asumir, también, sus expectativas ${ }^{47}$.
Se supone, asimismo, que una vez tomada la decisión los pacientes requieren saber todas las eventualidades de su tratamiento. En el genuino consentimiento informado se entiende que la información está destinada a orientar al paciente para que, con debidas razones, decida sobre las intervenciones propuestas, opte por alguna alternativa adicional que ha sido puesta en su conocimiento 0 , simplemente, rechace lo planteado o solicite más información. De modo que el proceso carece de rigideces o rutinas que deban tener una cronología invariable. Lo importante y decisivo es la información inteligible que, aunque compleja, pueda ser accesible a los pacientes mediante una comunicación facilitada por el interés de transmitírselas. No faltan quienes alegan que los pacientes son incapaces de entender las dificultades de los temas médicos; pero no es a eso lo que aspiran los enfermos y para lo que les sirve el consentimiento, sino que a comprender y hacer suyos los objetivos y propósitos de las intervenciones médicas que el profesional tiene en mente para controlar la enfermedad o el padecimiento que los afecta.

Otros dos supuestos, se refieren a que a los pacientes se les debe dar información, lo quieran o no, y a que ella se puede retener si se presume que pueda hacer rehusar el tratamiento, al paciente. Si recordamos que la información tiene por objetivo que el paciente, en virtud de su autonomía, tome la decisión que esté más de acuerdo con su proyecto de vida y en conformidad a los principios y valores bioéticos fundamentales, ambas suposiciones pueden descartarse al lograr una relación clínica empática y beneficiente. Incluso el privilegio terapéutico ${ }^{47,48}$ considerado también entre las excepciones al consentimiento informado, puede hacerse prescindible, si se obtiene un grado de confianza inembargable, del paciente por el médico, en la relación clínica.

Terminemos por mencionar que se puede establecer exención para el consentimiento informado, cuando con ello se trata de prevenir un grave riesgo para la salud pública o por razones de tipo legal o judicial. La consideración del "mal menor" suele ser, en todos estos casos, la justificación para una excepción a una información razonada que, de otra manera, es flagrante violación a la autonomía de los pacientes y a su dignidad personal ${ }^{45}$. 


\section{REFERENCIAS}

1. Los Objetivos Sanitarios para la Década 20002010. MINSAL. Gobiemo de Chile. Octubre, 2002.

2. Proyecto de Ley que establece un régimen de garantías en Salud [Plan de Acceso Universal con Garantías Explícitas (AUGE)] e indicaciones al proyecto (Boletín 2947-11), Mensajes de SE el Presidente de la República a la Honorable Cámara de Diputados, de 22 de mayo y 25 de junio de 2002, respectivamente.

3. Proyecto de Ley sobre los Derechos y Deberes de las Personas en Salud. Indicación Sustitutiva a la Honorable Cámara de Diputados (Boletín $\mathrm{N}^{\circ}$ 2727-11). Noviembre, 19, 2002.

4. Proyecto de Ley que modifica la Ley 18.933 sobre Instituciones de Salud Previsional Mensaje de SE el Presidente de la República a la Honorable Cámara de Diputados, 25 de junio de 2002.

5. Proyecto de Ley que establece el financiamiento del Gasto Fiscal representado por el Plan Auge y Chile Solidario. Mensaje de SE el Presidente de la República a la Honorable Cámara de Diputados, de 25 de junio de 2002.

6. Proyecto de Ley que modifica el Decreto Ley $\mathrm{N}^{\circ}$ 2.763 de 1979, sobre Gestión y Autoridad Sanitaria. Mensaje de SE el Presidente de la República a la Honorable Cámara de Diputados, del 25 de junio de 2002.

7. Rosselot E. Aspectos bioéticos comprendidos en la Reforma de la Atención de la Salud en Chile. I.Los problemas del acceso a la salud y el costo de los recursos. Rev Méd Chile 2003; 131: 1079-86.

8. Beauchamp TL, Childress JF. Principles of Biomedical Ethics. New York, Oxford University Press. $4^{\mathrm{a}}$ Ed, 1994.

9. Dougherty CJ. Ethical values at stake in health care reform. JAMA 1992; 268: 2409-12.

10. Rosselot E. Derechos del paciente, en el marco de la calidad de la atención médica. Rev Méd Chile 2000; 128: 904-10.

11. Rosselot E. El derecho a una atención médica de buena calidad. Rev Méd Chile 2000; 128: 1385-8.

12. KAss NE. An ethics framework for public health. Am J Public Health 2001; 91: 1776-82.

13. Daniels N, Kamm FM, Rakowski E, Broome J, Bailey MA. Meeting the challenges of justice and rationing. Hastings Center Report 1994; 24: 27-42.
14. Rawls J. Teoría de la Justicia. Madrid, FCE, 1979.

15. Gracia D. Profesión Médica, Investigación y Justicia Sanitaria. El Buho, Bogotá, Colombia, 1998.

16. Fiscella K, Franks P, Gold MR, Ciancy CM. Inequality in quality. Addressing socioeconomic, racial and ethnic disparities in health care. JAMA 2000; 283: 2579-84.

17. LeNoIR N. Bioética, constituciones y derechos humanos. Cuad Méd Soc 1997; 38: 3-14.

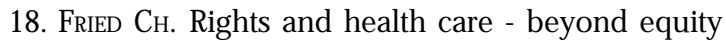
and efficiency. N England J Med 1975; 293: 241-5.

19. MINSAL Diseño e implementación de las prioridades de salud. La Reforma Programática Chilena. DISAP, 1997.

20. Salinas H, Lenz, Herrera G, Bustos P, Garín F. Las NO reformas de salud en Latinoamérica, razones que explican su fracaso. Andros, Santiago, Chile 2001.

21. CADORÉ B. L'expérience bioéthique de la responsabilité. Catalyses. Artel/Fides. Belgique/Montreal. 1994.

22. Council on Ethical and Judicial Affairs. Ethical issues involved in the growing AIDS crisis. JAMA 1988; 259: 160-1.

23. van den Burg PJ, VRIELINK H, ReEsink HW. Donor selection: the exclussion of high risk donors? Vox Sang 1998; 74 (Suppl 2): 499-502.

24. SICARD D. The precaution principle and blood transfusion. Transfus Clin Biol 2000; 7: 220-7.

25. SAGREDO F. La reforma acaba con la libre elección. Vida Médica 2002; 54: 20-3.

26. Project of the ABIM Foundation, ACP - ASIM Foundation, and European Federation of Internal Medicine. Medical professionalism in the new millenium: A Physician Charter. Ann Intern Med 2002; 136: 243-6. (Rev Méd Chile 2003; 131: 457-60).

27. Rosselot E. Reivindicando la medicina como profesión científico humanista. Rev Méd Chile 2003; 131: 454-5.

28. ReLMAN AS. Dealing with conflict of interest. N Engl J Med 1985; 313: 749-51.

29. Council on ETHICAL AND JUDICIAL AFFAIRES. AMA. Conflicts of Interest. Physician ownership of medical facilities. JAMA 1992; 267: 2366-9.

30. De los Reyes LM, Pérez GJM, García OP, Borrell CF, Gracia GD. Relaciones entre profesionales sanitarios. Med Clín (Barc) 2001; 117: 339-50.

31. CaLdahan D. Medicine and the markett. A research agenda. J Med Philos 1999; 24: 224-42. 
32. Swick HM. Academic Medicine must deal with the clash of business and professional values. Acad Med 1998; 73: 751-5.

33. CoHEN JJ. Protecting consumers or caring for patients? Acad Med 1998; 73: 878-9.

34. Publicidad y Profesión Médica. Comisión de Etica. Colegio Médico de Chile AG. 07/2003.

35. Grumbach K, Selby JV, Damberg C et al. Resolving the gatekeeper conundrum. JAMA 1999; 282: 2616.

36. Simón P. La ética de las organizaciones sanitarias: el segundo estadio de desarrollo de la bioética. Rev Calidad Asistencial 2002; 17: 242-59.

37. Hiatt HH. Protecting the medical commons. N Engl J Med 1975; 293: 235-41.

38. Olweny $\mathrm{CH}$. Bioethics in developing countries: ethics of scarcity and sacrifice. J Med Ethics 1994; 20: $169-74$.

39. MiL JS. El utilitarismo. Alianza Ed. Madrid. 1991.

40. Gracia D. Fundamentación y enseñanza de la bioética. El Buho. Bogotá. Colombia. 1998.
41. Protocolo Derechos del Paciente. Fondo Nacional de Salud. Ministerio de Salud. República de Chile. 1999.

42. BAezA H. Consentimiento informado. Rev Méd Chile 1995; 123: 1525-8.

43. Centro de Ética. Universidad Alberto Hurtado. Consentimiento informado: iprotección, favor, derecho! Informe Ethos 2001; 15.

44. Código de Nuremberg: Normas éticas sobre experimentación en seres humanos. Nuremberg, 20 de agosto de 1947.

45. Simón P, Júdez J. Consentimiento informado. Medic Clín (Barc) 2001; 117: 99-106.

46. GaMBLE VN. Under the shadow of Tuskegee: African Americans and health care. Am J Publ Health 1997; 87: 1773-8.

47. MeIsel A, KucZEwsKi M. Legal and ethical myths about informed consent. Arch Int Med 1996; 156: 2521-6.

48. Gracia D. Bioética Clínica. El Buho. Bogotá. Colombia. 1998. 